\title{
The changing face of oral anticoagulants
}

\author{
O. J. Rider*1 and E. B. Rider ${ }^{2}$
}
IN BRIEF
- Enables readers to gain a better understanding of the evidence behind the new generation of oral anticoagulants.
- Allows readers to gain a familiarity with the action and pharmacokinetics of the newer anticoagulants with comparison to warfarin.
- Provides current guidelines for management of bleeding.

\begin{abstract}
Warfarin has been the established oral anticoagulant for the last 50 years, being effective in the prevention and treatment of venous and arterial thromboembolic disorders. However, the frequent requirement for INR monitoring, multiple drug and food interactions have fuelled the need for development of new oral anticoagulants. Dabigatran is the first of a series of new oral anticoagulants that are emerging as the successors to warfarin. This new group of anticoagulants is rapidly gaining FDA and NICE approval and has proven non-inferiority to warfarin and viable alternatives to warfarin in the coming years. Given the obvious impact of this on dental treatment in the primary care and hospital setting this article aims to increase familiarisation with this new medicine group.
\end{abstract}

\section{INTRODUCTION}

Although warfarin was initially marketed as a rodent pesticide in 1948 (and is still used for this purpose) by 1954 it was found to be effective and relatively safe in the prevention at treatment of venous and arterial thromboembolic disease. ${ }^{1}$ Warfarin is still, over 50 years later, the most widely prescribed oral anticoagulant in the United Kingdom bibliography ${ }^{2,3}$ and remained, until recently, the only licensed drug for long-term anticoagulation. However, despite this, warfarin has several drawbacks which have prompted the search for newer, safer anticoagulants. Dabigatran etixilate (Pradaxa) is the first of a new generation of oral anticoagulants which are now available on the anticoagulant market. Dental surgeons in the primary care setting and those in the secondary hospital care setting will very soon be faced with decisions on the safety of surgical procedures on patients in whom these new anticoagulants are present. As they are pharmacologically distinct from

${ }^{1 *} 0 x f o r d$ Centre for Clinical Magnetic Resonance Research, Department of Cardiovascular Medicine, University of Oxford; ${ }^{2}$ Oasis Dental Centre, Wantage, Oxfordshire

*Correspondence to: Dr Oliver Rider

Email: oliver.rider@gmail.com

\section{Refereed Paper}

Accepted 8 May 2013

DOI: 10.1038/sj.bdj.2013.628

${ }^{\circledR}$ British Dental Journal 2013; 215: 17-20 warfarin, they have a different side-effect profile and have raised concern in regards to bleeding complications. This article is aimed at providing a background to the pharmacology, timing or surgery and management of complications of these new drugs which are now available alternatives to warfarin. The focus of this review is dabigatran etixilate as it has recently achieved NICE approval ${ }^{4}$ but the other newer agents (rivaroxaban and apixaban [both factor Xa inhibitors]) will also be considered. ${ }^{5,6}$

\section{THE RATIONALE FOR CHANGE}

Although several anticoagulants are currently available, some are not available in oral formulation (heparin, low molecular weight heparins, fondaparinux) and, as such, are not suitable for the majority of patients requiring long-term anticoagulation for the chronic management of thromboembolism. Warfarin, however, is an oral preparation that has been shown to be effective in the prevention, as well as treatment, of various thromboembolic disorders. As a result of this, and the relatively long half-life (mean of around 40 hours) allowing a once daily dosing regimen, warfarin has in the United Kingdom over the last 50 years been used almost exclusively for long-term anticoagulation. Despite this, warfarin has several disadvantages which include an increased risk of bleeding, slow onset of action, and a narrow therapeutic window. In addition, the significant inter-individual variability of action and multiple drug and food interactions result in an unpredictable level of anticoagulation with low levels of 'in target range'. Given the potential for bleeding with over-anticoagulation and thromboembolism with under-anticoagulation, frequent international normalised ratio monitoring (INR) measurements are required.

As a direct result of this, a new generation of oral anticoagulants has been developed to treat and prevent thromboembolic disorders, 'direct thrombin inhibitors' and the factor Xa inhibitors. The first generation of these drugs (bivalrudin, argatroban and the now UK discontinued lepirudin), although effective, have to be given intravenously and therefore were reserved primarily for inpatient treatment of heparin-induced thrombocytopenia and in patients undergoing percutaneous coronary interventions. However, the second generation of these drugs is now available in oral preparations, and are well placed to supersede warfarin therapy.

\section{THE EVIDENCE FOR NEW ORAL ANTICOAGULANTS}

Dabigatran etexilate (Pradaxa, Boehringer Ingelheim) is one of the new oral preparations of direct thrombin inhibitors and is 
the first one of its kind to be available in the United Kingdom. In March 2012, dabigatran etexilate received NICE approval as an alternative to warfarin for risk reduction in stroke and systemic embolism in patients with nonvalvular atrial fibrillation. In a non-inferiority trial of 18,000 patients with nonvalvular atrial fibrillation, the primary outcome of stroke or systemic embolism was similar in those taking dabigatran to those taking warfarin at $1.69 \%$ per year in the warfarin group, as compared with $1.53 \%$ per year in the group that received $110 \mathrm{mg}$ of dabigatran (relative risk with dabigatran, 0.91; 95\% confidence interval [CI], 0.74 to 1.11 ; $\mathrm{P}<0.001$ for noninferiority) and 1.11\% per year in the group that received $150 \mathrm{mg}$ of dabigatran (relative risk, 0.66; 95\% CI, 0.53 to 0.82 ; $\mathrm{P}<0.001$ for superiority). Importantly it was associated with a $32 \%$ relative risk reduction of major bleeding (3.36\% per year in the warfarin group, as compared with $2.71 \%$ per year in the group receiving $110 \mathrm{mg}$ of dabigatran $[\mathrm{P}=0.003]$ and 3.11\% per year in the group receiving $150 \mathrm{mg}$ of dabigatran $[\mathrm{P}=0.31]){ }^{8}$

Two other oral direct thrombin inhibitors have also been developed rivaroxaban (Xarelto) and apixiban (Eliquis) ${ }^{9}$ and also have data in regards to thromboembolic prevention. ${ }^{10-13}$ Again the rate of major bleeding was lower in those taking rivaroxaban and apixiban than those in the warfarin group.

These trials pave the way for widespread prescription and given the high prevalence of atrial fibrillation in the general population this has obvious implications for out of hospital dental surgical procedures. Where traditionally dental surgeons would be familiar with warfarin pharmacokinetics and baseline INR levels at which surgical procedures and injections would be relatively safe, the pharmacology of the newer direct thrombin inhibitors will make this a more difficult prospect. Due to the fact that dabigatran, rivaroxaban and apixaban have a predictable anticoagulation response, a fast onset and offset of action, and are not affected by dietary factors, they do not require routine anticoagulation monitoring. Although this can be seen as a major advantage over warfarin, there is no current widely available method of determining the exact level of anticoagulation (the INR is not a reliable

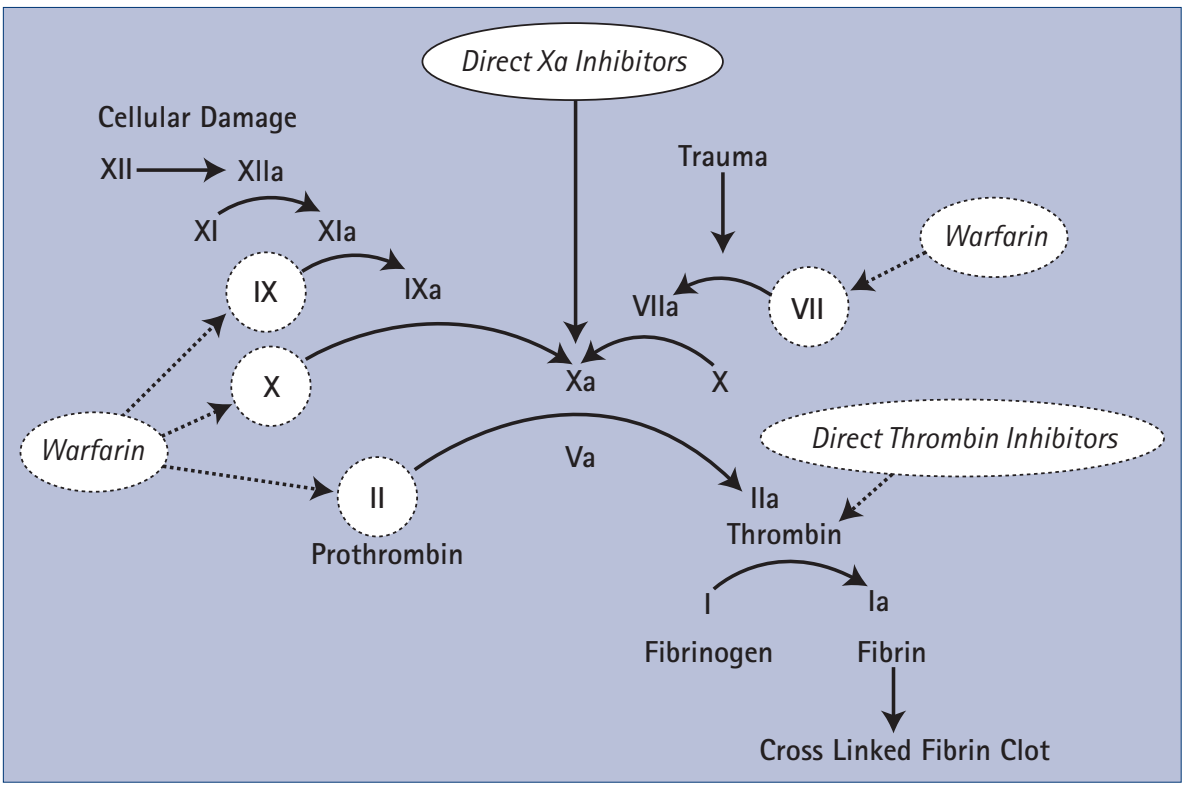

Fig. 1 The target of anticoagulation with vitamin $\mathrm{K}$ antagonist, warfarin and direct thrombin inhibitors

indicator of the level of anticoagulation with direct thrombin inhibitors) and certainly in the United Kingdom experience with surgical extraction while on these new drugs is very limited and will require a significant shift in the current practice of pre-surgical INR checks.

\section{PHARMACOLOGY OF DIRECT THROMBIN INHIBITORS AND FACTOR XA INHIBITORS}

Unlike warfarin, which acts via inhibiting the vitamin $\mathrm{K}$ dependent synthesis of clotting factors II, VII, IX and X (Fig. 1) which then inhibits the downstream formation of fibrin clots, the direct thrombin inhibitors act directly on thrombin, a plasma serine protease which plays a key role in the formation of clot. Thrombin primarily acts to convert fibrinogen to fibrin. In view of the fact that platelet activation and aggregation is most physiologically stimulated by thrombin, the inhibition of thrombin is an ideal mode of action for an anticoagulant agent (Fig. 1).

Dabigatran etexilate is a prodrug which is absorbed in the gastrointestinal tract. Once absorbed, dabigatran etexilate is rapidly and almost completely hydrolysed into two intermediates, BIBR 1087 and BIBR 951, by esterases found in the plasma and liver. These products are further metabolised to the final active agent, dabigatran. As the cytochrome P450 system, a source of many drug interactions, is not involved in the processing of dabigatran etexilate, the variation of metabolism with food and most other drugs is removed.

Elimination of dabigatran after hepatic activation occurs predominantly (up to 80\%) in the kidneys. Dabigatran's rapid onset of action results in peak serum levels approximately two hours after oral dosing. Once at steady state, the terminal half-life is 1,318 hours (prolonged if renal function is impaired); see Table 1 .

Due to the fact that dabigatran is a substrate for the efflux transporter Pgp, its plasma concentrations are affected by concomitant administration of Pgp inhibitors such as amiodarone, verapamil, quinidine, ketoconazole, dronedarone and clarithromycin and Pgp inducers such as rifampicin, St. John's wort (Hypericum perforatum), carbamazepine, or phenytoin. ${ }^{14}$

Rivaroxaban and apixaban are both direct factor Xa inhibitors, ${ }^{15}$ whose rapid onset of action results in peak serum levels approximately three hours after oral dosing. Once at steady state, the terminal half-life is four to nine hours (up to 12 hours in patients $>75$ years old). Rivaroxaban inhibits both the 'free' and prothombinase-complex-bound forms of activated factor X. As around 65\% percent of rivaroxaban is renally excreted caution is advised in severe renal failure. Apixaban, in addition to 25\% renal excretion, is metabolised in part by CYP3A4 in the liver. Rivaroxaban and Apixaban are not recommended in patients receiving concomitant systemic treatment with 
azole-antimycotics such as ketoconazole, itraconazole, voriconazole and posaconazole or HIV protease inhibitors. These active substances are strong inhibitors of both CYP3A4 and Pgp. Active substances strongly inhibiting only one of the anticoagulant's elimination pathways, either CYP3A4 or Pgp, are expected to increase plasma concentrations to a lesser extent such, as Clarithromycin. Strong CYP3A4 inducers should be co-administered with caution as well. ${ }^{16,17}$

\section{NECESSARY CHANGES IN THE MANAGEMENT OF SURGERY ON ANTICOAGULATION}

The management of bleeding complications in patients on the newer direct thrombin inhibitors has raised concern for clinicians and given the fact that dental surgeons will soon operate on patients anticoagulated with these newer drugs this is likely to remain a concern. The majority of concern with the direct thrombin inhibitors lies in the fact that, unlike warfarin, where vitamin $\mathrm{K}$ is an effective reversal agent, no effective antagonist is currently available.

Current practice with warfarin is that INR should be measured and recorded in the patient's notes no more than 72 hours before extractions, sub gingival scaling, infiltrations or administration of an inferior dental block. ${ }^{18-20}$ In addition, patients with unstable INRs should be assessed within 24 hours of the dental procedure. As it is likely that the risk of significant bleeding in patients on oral anticoagulants and with a stable INR in the therapeutic range of 2.0-4.0 (ie less than 4.0) is very small, and the risk of thrombosis may be increased in patients in whom oral anticoagulants are temporarily discontinued, it is recommended that oral anticoagulants should not be discontinued in the majority of patients requiring outpatient dental surgery including dental extraction.

Despite this, there is specific guidance. Patients with an INR over 4.0 should be referred to their anticoagulation service. The procedure rescheduled when the INR is less than 4.0, or referred to specialist services if their INR remains above 4.0 or control, is erratic. Patients with an INR of 4.0 or below should have the timing of the dental procedure considered. It is recommended treatment takes place in the morning at the

Table 1 Current guidelines for the cessation of dabigatran pre-operatively

\begin{tabular}{|c|c|c|c|}
\hline \multirow{2}{*}{$\begin{array}{l}\text { Renal function } \\
\text { ( } \mathrm{CrCL} \text { in } \\
\mathrm{mL} / \mathrm{min} \text { ) }\end{array}$} & \multirow{2}{*}{$\begin{array}{l}\text { Estimated } \\
\text { half-life } \\
\text { (hours) }\end{array}$} & \multicolumn{2}{|l|}{ Stop dabigatran before elective surgery } \\
\hline & & High risk of bleeding or major surgery & Standard risk \\
\hline$\geq 80$ & $\sim 13$ & 2 days before & 24 hours before \\
\hline$\geq 50<80$ & $\sim 15$ & $2-3$ days before & 1-2 days before \\
\hline$\geq 30<50$ & $\sim 18$ & 4 days before & $2-3$ days before ( $>48$ hours) \\
\hline
\end{tabular}

beginning of the week when re-bleeding problems can be managed during the working day and working week.

A local anaesthetic containing a vasoconstrictor should be used, where possible an infiltration, intraligamentary or mental nerve injection. If there is no alternative and an inferior alveolar nerve block is used, the injection should be administered slowly using an aspirating technique.

It is also recommended that after extraction the socket should be gently packed with an absorbable haemostatic dressing eg oxidized cellulose (Surgicel), collagen sponge (haemocollagen) or resorbable gelatin sponge and sutured and pressure applied using a gauze pad that the patient bites down on for 15-30 minutes. The patient should be provided with written post extraction instructions, additional swabs to take home and an emergency contact number in case of excessive bleeding and should not be co-prescribed NSAIDS or COX2 inhibitors. Should severe bleeding occur, vitamin $\mathrm{K}$ can always be administered to reverse the anticoagulation.

However, these current recommendations for warfarin are insufficient to supply full guidance for dental surgeons operating on patients in whom direct thrombin inhibitors or factor Xa inhibitors are being prescribed.

Although this article is not intended to provide clinical guidelines, the pharmacology of the newer anticoagulant agents should allow general guidance to be made, and existing guidelines from other surgical specialties can be adapted.

\section{TIMING OF SURGERY ON DIRECT THROMBIN INHIBITORS}

Given the rapid on/rapid off pharmacokinetics of the newer drugs, cessation before surgery and then restarting the drug after surgery is likely to be the way in which safe elective dental surgery will be performed. The half-life of these drugs is shorter than warfarin (at 12 to 17 hours for dabigatran) $)^{21}$ and is in the main related to renal function, with longer drug half-life occurring with worsening renal function. There is already guidance use of dabigatran in other surgical settings which can be adapted to the dental setting (Table 1).

As with warfarin, assessment of the risk of bleeding against the risk of thrombosis should be considered when discontinuing anticoagulation. It is likely that for nonsurgical dental procedures that dabigatran does not need to be discontinued (prosthodontics, conservation and endodontics). However, for surgical dental procedures, (such as extractions, periodontal surgery, minor oral surgery and biopsies, or where bleeding is expected), it will be important to plan ahead as there is no currently available treatment to reverse the action of dabigatran (and the other direct thrombin and Xa inhibitors).

Although impractical for the primary care setting, for hospital treatment, renal function should be checked at the preadmission clinic (or on admission) as this will have a major impact on when to stop the drugs before surgery ${ }^{22}$ (Table 1). It will be vital for all dentists to become familiar with when to stop these drugs and more importantly to provide clear instructions to patients about when to stop them.

It is currently unclear if it will become necessary for patients with normal renal function, undergoing elective dental surgery with low risk of bleeding to stop dabigatran for the preceding 24 hours. However, it would seem likely that given the very small increased risk of thrombosis associated with stopping anticoagulation for a 24 hour period, and the fact that reanticoagulation is very quick due to the rapid on kinetics, and additionally the lack of a reversal agent, this approach would be appropriate. 


\section{MANAGEMENT OF BLEEDING}

However, clear and specific advice in regards to bleeding will need to be documented for all patients and clinicians in regards to the potential for irreversible bleeding. Current recommendations from manufacturers include supportive measures with pressure, activated charcoal if drug ingestion was within a couple of hours, and haemodialysis if feasible for dabigatran, but given the fact that bleeding is due to clotting factor inhibition and not depletion the additional administration of clotting factors is unlikely to be helpful. ${ }^{23}$ The mainstay of reversal is time dependent renal excretion, which in the setting of renal insufficiency is significantly delayed. It is clear that any emergency surgery in the primary or hospital setting will require training and protocols to be in place to deal with unexpected bleeding.

Despite Boehringer Ingelheim recently writing to all dentists with product information on Dabigitran (August 2012), this was somewhat lacking, and there is currently no accessible UK data to help inform how best patients on the new oral anticoagulants should be managed when they require dental treatment.

\section{PROPOSED MANAGEMENT RECOMMENDATIONS OF PATIENTS TAKING DABIGATRAN IN THE PRIMARY DENTAL SETTING}

Assess dental procedure planned and risk of bleeding. If 'standard' surgical risk (extraction, minor oral surgery, periodontal surgery, biopsy), consult anticoagulation service. Dabigatran should be temporarily discontinued before the procedure is carried out in accordance with the patient's renal function.

Minimisation of risk should be undertaken in accordance with the recommendations for the treatment of patients on warfarin medication (timing of procedure, local anaesthetic used and post-op dressings).

\section{CONCLUSION}

Emerging clinical data have recently prompted NICE to recommend dabigatran 'as an option for the prevention of stroke and systemic embolism within its licensed indication, that is, in people with nonvalvular atrial fibrillation' and two other factor Xa inhibitors, rivaroxaban and apixaban, are likely to follow. These newer agents have different pharmacology and reversibility to the historical alternative of warfarin. Dental surgeons are likely to be operating on patients who are anticoagulated with these newer agents in the very near future. As such a widespread effort in education around surgery while on direct thrombin inhibitors, and protocols for bleeding management are needed.

1. Link K P. The discovery of dicumarol and its sequels. Circulation 1959; 19: 97-107.

2. Wadelius M, Pirmohamed M. Pharmacogenetics of warfarin: current status and future challenges. Pharmacogenomics J 2007; 7: 99-111.

3. Kamali F, Pirmohamed M. The future prospects of pharmacogenetics in oral anticoagulation therapy. Br J Clinical Pharmacol 2006; 61: 746-751.

4. Ahmad Y, Lip G Y. Dabigatran etexilate for the prevention of stroke and systemic embolism in atrial fibrillation: NICE guidance. Heart 2012; 98: 1404-1406.

5. Apixaban for preventing stroke and systemic embolism in people with nonvalvular atrial fibrillation. NICE Technology Appraisal 275, 2013

6. Venous thromboembolism (treatment and long term secondary prevention) rivaroxaban. NICE Technology Appraisal TA261, July 2012.

7. Onundarson $P$ T, Einarsdottir K A, Gudmundsdottir $B$ R. Warfarin anticoagulation intensity in specialist-based and in computer-assisted dosing practice. Int J Lab Hematol 2008; 30: 382-389.

8. Connolly S J, Ezekowitz M D, Yusuf S et al. Dabigatran versus warfarin in patients with atria fibrillation. N Engl J Med 2009; 361: 1139-1151.

9. Lassen M R, Raskob G E, Gallus A, Pineo G, Chen D, Portman R J. Apixaban or enoxaparin for thromboprophylaxis after knee replacement. N Eng / J Med 2009; 361: 594-604.

10. Lassen M R, Ageno W, Borris L C et al. Rivaroxaban versus enoxaparin for thromboprophylaxis after total knee arthroplasty. N Engl J Med 2008; 358: 2776-2786.

11. Eriksson B I, Borris L C, Friedman R J et al. Rivaroxaban versus enoxaparin for thromboprophylaxis after hip arthroplasty. N Eng/ J Med 2008; 358: 2765-2775.

12. Turpie A G, Lassen M R, Davidson B L et al. Rivaroxaban versus enoxaparin for thromboprophylaxis after total knee arthroplasty (RECORD4): a randomised trial. Lancet 2009; 373: 1673-1680.

13. Kakkar A K, Brenner B, Dahl O E et al. Extended duration rivaroxaban versus short-term enoxaparin for the prevention of venous thromboembolism after total hip arthroplasty: a double-blind, randomised controlled trial. Lancet 2008; 372: 31-39.

14. Boehringer ingelheim. Pradaxa ${ }^{\circledR} 110 \mathrm{mg}$ hard capsules summary of product characteristics.

15. Granger C B, Alexander J H, McMurray J J et al. Apixaban versus warfarin in patients with atrial fibrillation. N Engl J Med 2011; 365: 981-992.

16. Bristol-Myers Squibb-Pfizer Eliquis $2.5 \mathrm{mg}$ filmcoated tablets Summary of Product Characteristics.

17. Bayer Xarelto $20 \mathrm{mg}$ film-coated tablets. Summary of product characteristics.

18. Thromboembolic disease. Online information available at www.medicinescomplete.com/mc/ bnf/current/PHP164thromboembolicdisease.htm (subscription required).

19. Dewan K, Bishop K, Muthukrishnan A. Management of patients on warfarin by general dental practitioners in South West Wales: continuing the audit cycle. Br Dent J 2009; 206: E8; discussion 214-215.

20. Perry D J, Noakes T J, Helliwell P S. Guidelines for the management of patients on oral anticoagulants requiring dental surgery. Br Dent J 2007; 203: 389-393.

21. Clemens A, Haertter S, Friedman J et al. Twice daily dosing of dabigatran for stroke prevention in atrial fibrillation: a pharmacokinetic justification. Curr Med Res Opin 2012: 28: 195-201.

22. Clemens A, van Ryn J, Sennewald R et al. Switching from enoxaparin to dabigatran etexilate: pharmacokinetics, pharmacodynamics, and safety profile. Eur J Clin Pharmaco/ 2012; 68: 607-616.

23. Kaatz S, Kouides P A, Garcia D A et al. Guidance on the emergent reversal of oral thrombin and factor Xa inhibitors. Am J Hematol 2012 87 Suppl 1: S141-S145. 